\title{
Effect of simulated mechanical recycling processes on the properties of poly(lactic acid)
}

\author{
F.R. Beltrán ${ }^{1}$, V. Lorenzo ${ }^{1}$, J. Acosta ${ }^{2}$, M.U. de la Orden ${ }^{1}$, J. Martínez-Urreaga ${ }^{1}$.
}

1 Dpto. Ingeniería Química Industrial y Medio Ambiente, Grupo "Polímeros, Caracterización y Aplicaciones", Universidad Politécnica de Madrid, E.T.S.I. Industriales, Madrid, 28006, Spain.

2 Dpto. Mecánica, Química y Diseño Industrial, Universidad Politécnica de Madrid, E.T.S.I. Diseño Industrial, Madrid, 28012, Spain.

Presenting author information:

e-mail: f.beltran@alumnos.upm.es

\begin{abstract}
The aim of this work is to study the effects of different simulated mechanical recycling processes on the structure and properties of PLA. A commercial grade of PLA was melt compounded and compression molded, then subjected to two different recycling processes, one of them comprising an accelerated aging and a second melt compounding and compression molding step, and other which included a demanding washing step after the accelerated aging process. The intrinsic viscosity measurements indicate that both recycling processes produce a degradation in PLA, which is more pronounced in the sample subjected to the washing process. DSC results suggest an increase in the mobility of the polymer chains in the recycled materials, however the degree of crystallinity of PLA seems unchanged. The optical, mechanical and gas barrier properties of PLA do not seem to be largely affected by the degradation suffered during the different recycling processes. These results suggest that, despite the degradation of PLA, the impact of the different simulated mechanical recycling processes on the final properties is limited. Thus, the potential use of recycled PLA in packaging applications is not jeopardized.
\end{abstract}

\section{Keywords}

Poly(lactic acid); mechanical recycling; optical properties; mechanical properties; gas barrier properties.

\section{Introduction}

Poly(lactic acid) is an aliphatic polyester produced, at industrial scale, by ringopening polymerization of lactide, a cyclic oligomer of lactic acid. The lactic acid is obtained from the fermentation of glucose, derived from agricultural sources such as corn, potato or sugar beet [1]. PLA present some interesting properties, such as its biodegradability, good processability, low cost production, low environmental impact and optical and mechanical properties similar to those of polystyrene or PET [2]. These good properties have caused a growing interest in PLA, making it one of the most important bioplastics in the market, specifically in packaging applications, with a global 
production capacity of 0.21 million tons in 2014. Furthermore, it is projected a sustained growth of the production of PLA, reaching the 0.5 million tons in 2020 [3].

This expected growth of the production, and consumption, of PLA could lead to some social and environmental problems in coming years. Firstly, the sustenance and health of poor countries could be threatened, as farmers could exchange the cheap food production for the high value crops used in the manufacture of PLA and others biopolymers [4]. Secondly, there is concern about the management of the wastes derived from the massive use of PLA, since the commercial grades used in packaging applications degrade at low rate in comparison with the accumulation of wastes [5]. These problems highlight the need to study the potential of extending the life of PLA based products, in order to reduce the consumption of essential raw materials and the generation of wastes.

There are several alternatives for the valorization of PLA wastes. Piemonte [6] carried out Life Cycle Analysis for PLA, evaluating four different final scenarios: incineration, composting, anaerobic digestion and mechanical recycling. The results showed that, from an environmental point of view, the best alternative for the valorization of PLA wastes is the mechanical recycling. This proposition is stronger if we consider the fact that global production capacity of PLA has exceeded the 200,000 tons, which is considered the critical mass for the mechanical recycling to be profitable [7]. However, there are still a number of issues that need to be addressed in order to evaluate the feasibility of the mechanical recycling of PLA. Firstly, PLA can contaminate other plastics present in the recycling stream, such as PET, decreasing the quality of the recycled products. Secondly, it is necessary to evaluate the cost of separation of PLA, and to develop a viable recovery system for this polymer. Finally, it is important to study the effect of the mechanical recycling on the performance of PLA, since if there is an important decrease of the properties of the recycled materials, the mechanical recycling would not be viable, compromising the low environmental impact of PLA [5, 8].

Although the relevance of the mechanical recycling of PLA is clear, to our best knowledge, there is little reported data about the effect of different mechanical recycling process on the performance of PLA. Pillin et al. [9] reported that after seven reprocessing cycles, a commercial grade of PLLA showed a decrease of $60 \%$ in the molecular weight, increased crystallization ability but no significant changes in the hardness and Young modulus measured by nanoindentation. Żenkiewicz et al. [10] found in their studies with PLLA, an important decrease of the tensile strength and thermal stability after 10 reprocessing cycles. Nascimento et al. [11] studied the effect of an extrusion process followed by an injection process in the structure and thermal and mechanical properties of PLA, concluding that a single reprocessing step does not have a significant impact on the performance of the polymer. These studies provide useful information about the effects of the thermomechanical degradation on some properties of PLA, however, they do not consider the degradation of PLA during its service life, and the potential impact it might have on the performance of the recycled polymer.

The main objective of this work is to study the effect of degradation in use and different simulated mechanical recycling processes on the structure and properties of PLA. A commercial grade of PLA, Ingeo 2003D, especially designed for packaging 
applications was melt processed by extrusion and then compression molded into films. Part of these films were subjected to two different recycling processes, one comprising an accelerated thermal and photochemical aging and a second extrusion and compression molding step, and other including a demanding washing before the second extrusion and compression molding step. The effect of these recycling processes was measured by means of dilute solution viscosimetry (IV), attenuated total reflectance infrared spectroscopy (FTIR-ATR), differential scanning calorimetry (DSC), thermogravimetric analysis (TGA), UV-Vis spectroscopy, haze measurements, instrumented nanoindentation tests and gas permeability measurements.

\section{Materials and methods}

\subsection{Material description and sample preparation}

A commercial grade of PLA designed for packaging applications (Ingeo 2003D, Natureworks $\left.{ }^{\mathrm{TM}}\right)$, with a melt mass-flow rate of $6 \mathrm{~g} / 10 \mathrm{~min}\left(2.16 \mathrm{~kg}\right.$ at $\left.210^{\circ} \mathrm{C}\right)$ was used. Prior to processing the pellets were crystallized, for 20 minutes at $100^{\circ} \mathrm{C}$, and dried in a vacuum oven at $85^{\circ} \mathrm{C}$ for $2 \mathrm{~h}$. Afterwards, the samples were processed by melt compounding in a Rondol Microlab twin-screw microcompounder, with $L / D=20$ and a screw speed of $60 \mathrm{rpm}$. The barrel temperature profile from hopper to die was $125,160,190,190,180{ }^{\circ} \mathrm{C}$.

The obtained materials were then molded by compression into films with a thickness of $230 \pm 10 \mu \mathrm{m}$ in a IQAP-LAP hot-plate press at $190{ }^{\circ} \mathrm{C}$, beginning with a melting step, with no pressure, for 5 minutes, followed by a degasification step for 2 minutes, and finally the samples were cooled between cold plates, using a pressure of $14 \mathrm{MPa}$ for 5 minutes.

In order to simulate the mechanical recycling of the polymer, the films of the virgin polymer were first subjected to an accelerated ageing process, which included 40 hours of photochemical degradation in an Atlas UVCON chamber, equipped with eight F40UVB lamps. The samples were also exposed to thermal degradation in a convection oven at $50^{\circ} \mathrm{C}$ for $468 \mathrm{~h}$. A portion of these aged samples was washed at 85 ${ }^{\circ} \mathrm{C}$ for 15 minutes, in a solution of $\mathrm{NaOH}$ ( $1.0 \%$ by weight) and a surfactant (Triton $\mathrm{X}$, $0.3 \%$ by weight). Finally, all aged samples were reprocessed by extrusion and compression molded into films, following the process explained above.

According to the recycling process there were three different materials: PLAV, which was not reprocessed, PLAR, which was recycled without the washing step, and PLARW which was recycled with the demanding washing step at $85^{\circ} \mathrm{C}$. Before the characterization, all the samples were subjected to physical aging, at room temperature, for 3 weeks.

\subsection{Characterization Techniques}

Infrared spectra of the different materials were recorded in a Nicolet iS10 spectrometer, equipped with an Attenuated Total Reflectance (ATR) accessory. Each spectrum was recorded at a resolution of $4 \mathrm{~cm}^{-1}$, with a total of 16 scans. The FTIR spectra were corrected using the software Omnic 9.2.41, and normalized using the band at $1450 \mathrm{~cm}^{-1}$, which has been reported as an internal standard [12]. 
The overall transmittance in the visible light region was measured according to the ISO 13468 standard, using a Shimadzu 2401 PC UV-Vis spectrophotometer equipped with a Shimadzu integrating sphere, using a scan speed of $200 \mathrm{~nm} / \mathrm{min}$. The haze of the different materials was measured according to the ISO 14782 standard, using a Minolta CMD3600D Spectrocolorimeter, with a diffuse $/ 8^{\circ}$ geometry. The value of the haze is given by the following expression:

$\%$ Haze $=\frac{\tau_{\mathrm{D}}}{\tau_{\mathrm{T}}} \times 100$

where $T_{D}$ is the diffuse transmittance and $T_{T}$ is the total transmittance.

The intrinsic viscosity (IV) was measured at $25 \pm 0.5^{\circ} \mathrm{C}$ using an Ubbelohde viscosimeter and chloroform as solvent.

Thermogravimetric analysis of the different samples was carried out using a TA Instruments TGA2050 thermobalance. Samples of $\approx 14 \mathrm{mg}$ were heated at $10{ }^{\circ} \mathrm{C} / \mathrm{min}$ from room temperature to $800{ }^{\circ} \mathrm{C}$ in dry nitrogen $\left(30 \mathrm{~cm}^{3} / \mathrm{min}\right)$.

Differential scanning calorimetry (DSC) analysis was performed on samples of about $7 \mathrm{mg}$, in standard aluminum pans, using a Mettler-Toledo DSC 823e calorimeter, under nitrogen atmosphere. The data was recorded at a heating rate of 10 ${ }^{\circ} \mathrm{C} / \mathrm{min}$. The samples were first heated from 20 to $220^{\circ} \mathrm{C}$, and then kept at $220^{\circ} \mathrm{C}$ for 3 minutes to erase thermal history. After that, the samples were cooled to $0^{\circ} \mathrm{C}$, and finally a second heating scan was performed until $220^{\circ} \mathrm{C}$. The crystallinity of PLA was determined by Eq. (2):

$\chi(\%)=\frac{\Delta \mathrm{H}_{\mathrm{M}}-\Delta \mathrm{H}_{\mathrm{CC}}}{\Delta \mathrm{H}_{\infty}} \times 100$

where $\Delta \mathrm{H}_{\mathrm{M}}$ and $\Delta \mathrm{H}_{\mathrm{CC}}(\mathrm{J} / \mathrm{g}$ of polymer) are the melting enthalpy and the cold crystallization enthalpy respectively. $\Delta \mathrm{H}_{\infty}$ is the melting enthalpy for a $100 \%$ crystalline PLA, it has a value of $93.1 \mathrm{~J} / \mathrm{g}$ [13].

The mechanical properties of the films were measured using a Shimadzu DUH$211 \mathrm{~S}$ dynamic Ultra-Microhardness Tester at room temperature. A maximum load of 10 $\mathrm{mN}$ and a loading rate of $1.4632 \mathrm{mN} / \mathrm{s}$ were selected. Maximum load was hold for $5 \mathrm{~s}$, and afterwards, load was retired. Each measurement was replicated 6 times.

The gas permeability of $\mathrm{N}_{2}, \mathrm{O}_{2}$ and $\mathrm{CO}_{2}$ was measured using a homemade permeation cell, with a gas pressure of 1 bar and at a temperature of $30^{\circ} \mathrm{C}$.

\section{Results and discussion}

\subsection{Degradation during the recycling processes}

It is known that PLA is susceptible to thermo-mechanical and hydrolytic degradation during melt processing. The polymer subjected to high temperatures and shear stresses could suffer chain scission processes, which cause a decrease in the 
molecular weight, and thus in the intrinsic viscosity. Besides the degradation during melt processing, PLA could also suffer degradation processes during its use. These processes can be thermal, photochemical and hydrolytic, and also cause a reduction in the molecular weight of the polymer [14].

In any case, the degradation of the polymer during its use and/or during its recycling could have an impact on the structure and on some properties such as thermal stability or mechanical and optical properties. Thus, it is very important to quantify the effect of the different recycling processes on the molecular weight of PLA. For this purpose, intrinsic viscosity measurements of all the samples were performed.

Fig. 1 shows the intrinsic viscosity values for the samples subjected to different recycling processes. It can be seen that the recycled PLA, without the washing step, presents a small decrease of the intrinsic viscosity in comparison with the virgin polymer (around $5 \%$ ). Similar results have been reported by other authors which reported marginal decreases in the molecular weight of PLA subjected to one reprocessing step $[9,15,16]$. The reduction of the intrinsic viscosity observed in our measurements, as it was said before, is due to the chain scission of PLA, as a result of the high temperatures and the shear stress to which the polymer was subjected during the reprocessing, and could also be attributed to the degradation during the accelerated aging. Nevertheless, the decrease of the intrinsic viscosity is small, so it can be expected that the effect of the mechanical recycling, in these conditions, on the mechanical, thermal and optical properties to be limited.

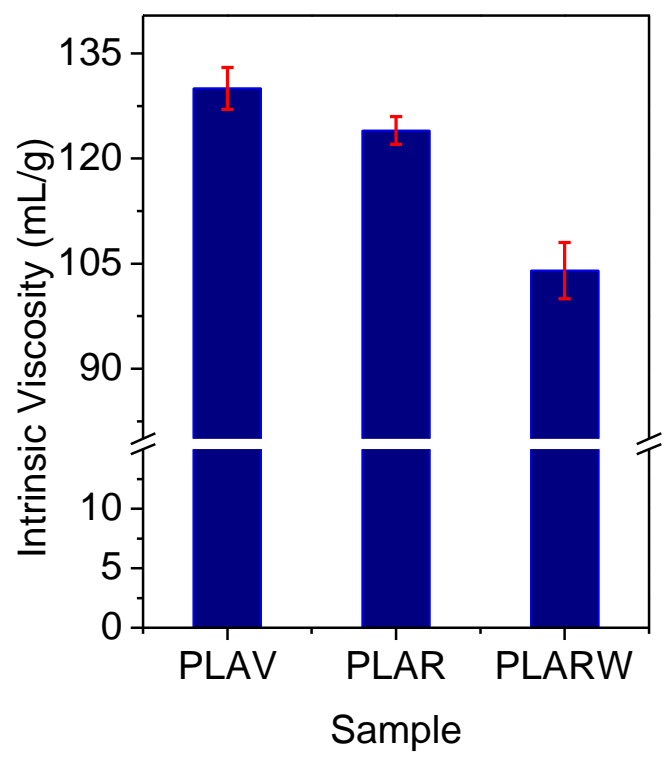

Fig. 1 Intrinsic viscosity values of PLA subjected to different simulated mechanical recycling processes

In the other hand, Fig. 1 also shows that PLARW presents a lower intrinsic viscosity than PLAV (20\% lower) and PLAR (16\% lower). This behavior suggest that the washing step plays an important role on the degradation of PLA during its mechanical recycling. 
In order to clarify this issue, intrinsic viscosity measurements were performed on PLA samples subjected to the washing step, but without being reprocessed by extrusion. The values of the intrinsic viscosity for these samples was $(123 \pm 1) \mathrm{mL} / \mathrm{g}$, which is higher than the value of the PLARW sample, $(104 \pm 5) \mathrm{mL} / \mathrm{g}$, and close to that of PLAV, $(130 \pm 3) \mathrm{mL} / \mathrm{g}$, suggesting that most of the degradation takes places during the reprocessing of the washed samples. Considering that all the samples were adequately dried prior to processing, this decrease of the intrinsic viscosity of PLARW suggests that the demanding washing process weakens the structure of the polymer, making it more susceptible to thermomechanical degradation.

Taking into account these results, we can conclude that PLA can withstand a single recycling process without suffering an important loss of molecular weight, however, the introduction of a washing step weakens the polymer structure, promoting the degradation during the reprocessing step. This reduction of the molecular weight could affect both the structure and final properties of PLA. Therefore, in the following sections we study the effect of the different recycling processes on the structure, thermal stability, and mechanical, optical and gas barrier properties of PLA.

\subsection{Monitoring of the structural changes by FTIR-ATR and DSC}

Once it has been analyzed the effect of the different recycling processes on the molecular weight of PLA, we studied the structural changes suffered by the polymer, which could have an impact on the mechanical, optical and gas barrier properties of the materials. Fig. 2 shows the FTIR-ATR spectra of the different samples, in the region between 3500 and $650 \mathrm{~cm}^{-1}$. The band assignment for PLA can be found in the literature [12]. As it can be seen, Fig. 2 does not show significant differences between the spectra of the three samples. Therefore, Fig. $\mathbf{3}$ show the enlargement of the area around $1750 \mathrm{~cm}^{-1}$. 


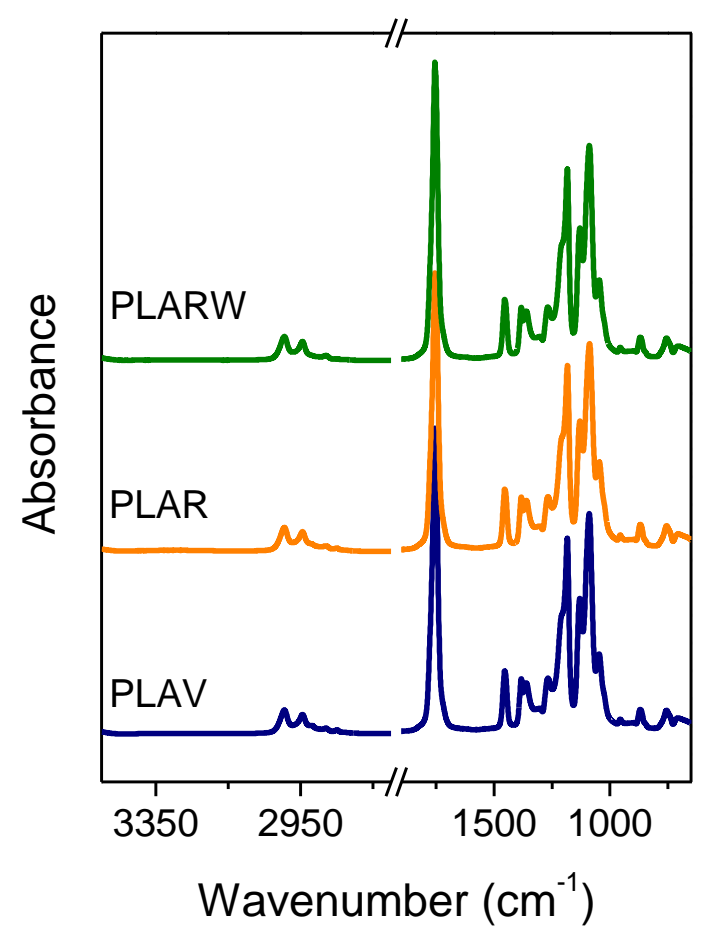

Fig. 2 FTIR-ATR spectra of PLA subjected to different simulated mechanical recycling processes

The absorption band centered at $1756 \mathrm{~cm}^{-1}$ corresponds to the stretching of the $\mathrm{C}=\mathrm{O}$ bonds of the ester groups originally present in PLA [12]. This band has been used as an indicator of the degradation of PLA, due to the generation of new carbonyl-linked species, both in the middle or in the end of the chains, during the reprocessing of PLA. In fact, Badia et al. [15] reported a displacement of the carbonyl stretching band towards higher wavenumbers as a consequence of several reprocessing steps. As it can be seen on Fig. 3, there are only minor differences between the virgin PLA and those subjected to the different mechanical recycling processes, thus revealing that only minor amounts of new carbonyl compounds are generated during the recycling of PLA. 


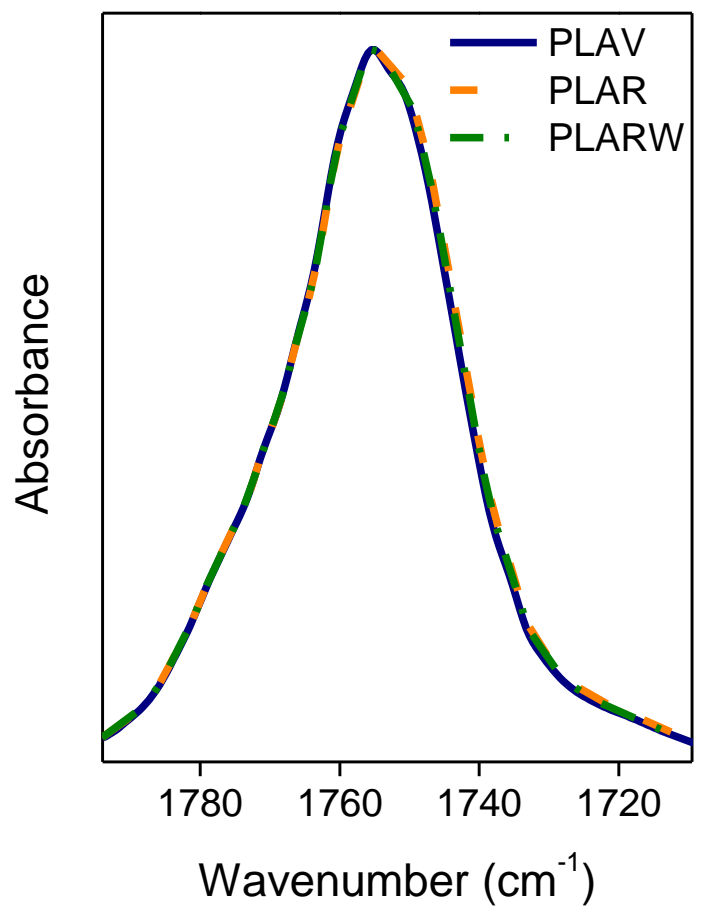

Fig. 3 Enlargement of the $\mathrm{C}=\mathrm{O}$ stretching band for the different samples

The changes in the structure of the polymer caused by the different recycling processes can be characterized using DSC. The first DSC heating scans of the different materials are presented on Fig. 4. It can be seen that all the samples present a glass transition around $60{ }^{\circ} \mathrm{C}$, accompanied by an endothermic peak related to the physical aging of PLA [11]. The recycling process does not seem to have a significant effect on the glass transition temperature $\left(T_{g}\right)$, calculated from the cooling DSC scans, of PLA (see also Table 1). 


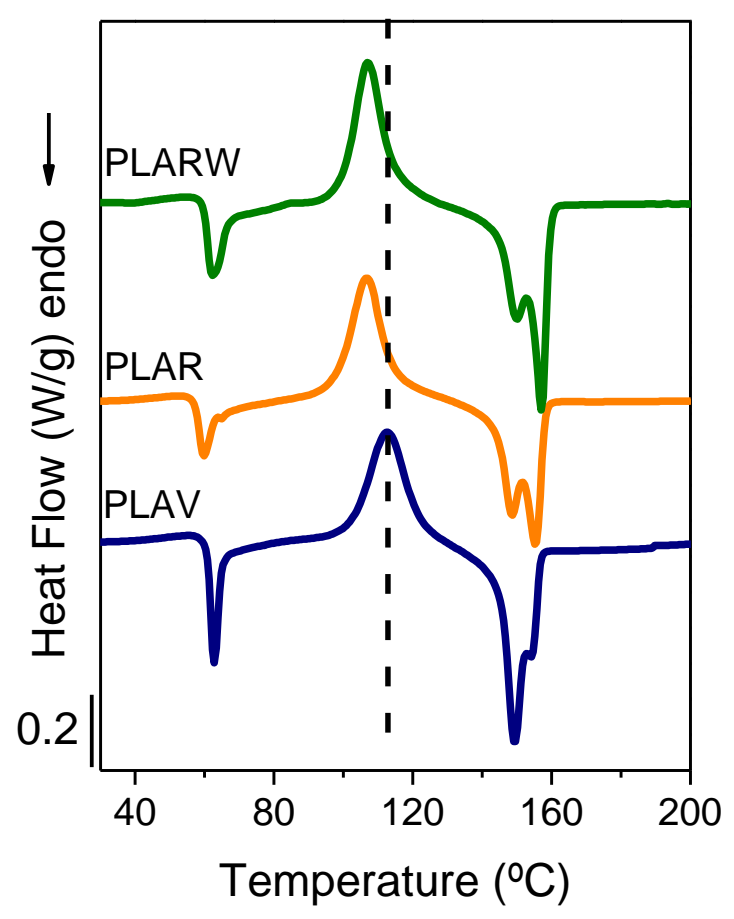

Fig. 4 First heating scans of PLA subjected to different simulated mechanical recycling processes

After the glass transition, there is an exothermic peak around $110{ }^{\circ} \mathrm{C}$, which corresponds to the cold crystallization of PLA [17]. It can be seen on Fig. 4 and on table 1 , that the recycled materials show a decrease of about $5{ }^{\circ} \mathrm{C}$ in the cold crystallization temperature $\left(T_{C C}\right)$. This reduction in the values of $T_{C C}$ has been attributed by Pillin et al. [9] and Scaffaro et al. [18] to a higher mobility of the polymer chains, as a consequence of the reduction of the molecular weight during the reprocessing. These results agree with those found by intrinsic viscosity measurements, where both PLAR and PLARW showed a decrease in the molecular weight, as a consequence of the mechanical recycling process.

Table 1 Results obtained from the DSC scans of the three PLA samples

\begin{tabular}{ccccccc}
\hline Sample & $\mathrm{T}_{\mathrm{G}}\left({ }^{\circ} \mathrm{C}\right)$ & $\mathrm{T}_{\mathrm{CC}}\left({ }^{\circ} \mathrm{C}\right)$ & $\mathrm{T}_{\mathrm{M} 1}\left({ }^{\circ} \mathrm{C}\right)$ & $\mathrm{T}_{\mathrm{M} 2}\left({ }^{\circ} \mathrm{C}\right)$ & $\Delta \mathbf{H}_{\mathrm{C}}(\mathrm{J} / \mathbf{g})$ & $\Delta \mathbf{H}_{\mathrm{M}}(\mathrm{J} / \mathbf{g})$ \\
\hline PLAV & 57.6 & 112.4 & 149.6 & 154.2 & 24.5 & 25.4 \\
PLAR & 57.1 & 106.9 & 148.7 & 155.1 & 23.2 & 24.7 \\
PLARW & 59.1 & 106.9 & 150.1 & 157.0 & 27.1 & 29.8 \\
\hline
\end{tabular}


Finally, a double endothermic peak appears around $150{ }^{\circ} \mathrm{C}$. This peak corresponds to the melting of the polymer. This double-melting behavior has been studied by several authors $[19,20]$, attributing it to a melt recrystallization mechanism, where the less perfect crystals melt at lower temperatures, reorganize in more stable crystals during the heating, and then melt at higher temperatures. According to table 1, there are no major differences in the melting temperatures of the three samples. However, there is a significant difference in the shape of the melting peaks between the virgin and the recycled materials. In PLAV, the low temperature melting peak $\left(T_{M 1}\right)$ is more relevant than the melting peak at high temperature $\left(T_{M 2}\right)$, while in PLAR and PLARW, the high temperature melting peak seems to increase its relevance. This difference could also be related with the decrease in the molecular weight of PLA, and its effect on the mobility of the polymer chains. During the melting, shorter polymer chains, as those present in the recycled materials, can effectively reorganize themselves into more ordered crystals, hence increasing the relevance of the high temperature melting peak. In the other hand, the polymer chains present in PLAV have limited mobility, due to their larger size, which prevents them to rearrange during the melting process.

Regarding the crystallinity of the different samples, we can see that, for each material, the crystallization and melting enthalpies are similar, suggesting that most of the crystals that melt during the heating are formed in the cold crystallization process. These results indicate that all the materials are amorphous, and that the recycling processes do not seem to cause any changes on the crystallinity degree of PLA, thus supporting the results obtained by means of FTIR-ATR spectroscopy. However, it is worth to note that, while PLAV and PLAR show similar values for $\Delta \mathrm{H}_{\mathrm{C}}$ and $\Delta \mathrm{H}_{\mathrm{M}}$, PLARW showed slightly higher values. This difference is related to the degradation of the previously washed PLA during the mechanical recycling, since, shorter polymers chains are able to crystallize more easily and, therefore, a bigger amount of crystals are formed. These results are in good agreement with those found by means of intrinsic viscosity measurements, where PLAR showed a molecular weight close to PLAV, while PLARW showed significant decrease of the intrinsic viscosity.

\subsection{Effect of the recycling processes on the properties of the materials}

\subsubsection{Thermal Stability}

After studying the degradation of PLA during the recycling processes, and the changes it causes on the structure of the polymer, we analyzed the effect of such recycling processes on some of the properties of PLA. The thermal stability was studied by means of TGA, and the obtained results are shown in Fig. 5, which presents the TGA curves for the three samples.

It can be seen on Fig. 5 that there is no significant difference between the TGA curves of PLAV and PLAR. This result indicates that the small reduction on the molecular weight that took place during the recycling process does not have a significant impact on the thermal stability of the sample. 


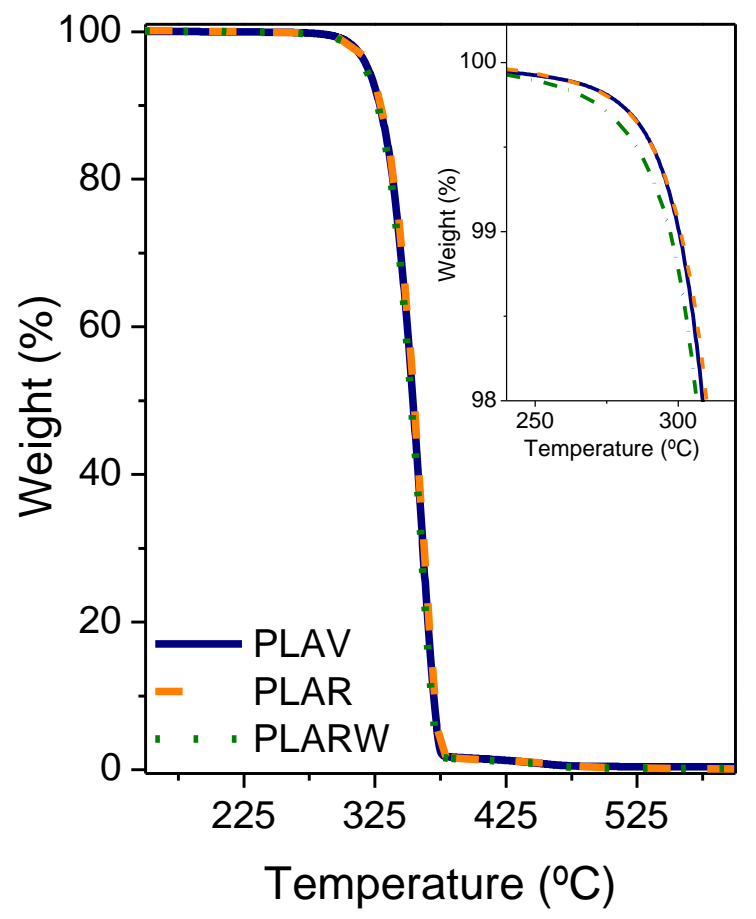

Fig. 5 TGA curves of PLA subjected to different simulated mechanical recycling processes

On the other hand, PLARW have a slightly lower thermal stability, which is related to the reduction in the molecular weight observed in the intrinsic viscosity measurements. It has been reported that the generation of shorter polymer chains with lower thermal stability is responsible for this perceived difference in the early stages of thermal degradation [21].

\subsubsection{Optical properties}

The results shown in the previous sections indicate that the recycling processes cause a reduction in the molecular weight and structural changes in PLA. These changes could have a negative effect on some optical properties of the materials, such as haze and light transmission, which play an important role in the applications of PLA in the packaging sector.

Fig. 6 shows the UV-Vis spectra of the different materials. It can be seen that there is an important difference in the absorbance of the samples between 250 and $300 \mathrm{~nm}$. In this range, the absorbance was increased in both PLAR and PLARW. The material subjected to the washing step presents an absorption band around $270 \mathrm{~nm}$. The higher UV-light absorption of the PLA recycled samples could be caused by the formation of hydroxyl end groups as a consequence of the chain scissions from thermo-mechanical degradation that took place during the recycling, as it was stated by Chariyachotilert et al. [16]. Furthermore, Badia et al. [22] observed that several reprocessing steps caused a change in the oligomeric distribution of PLA, increasing the amount of $-\mathrm{COOH}$ terminal groups. These results agree with those found by 
viscosimetry, where PLARW showed a larger decrease in the molecular than PLAR, due to the weakening of the polymer during the washing step, making it more susceptible to the thermo-mechanical degradation.

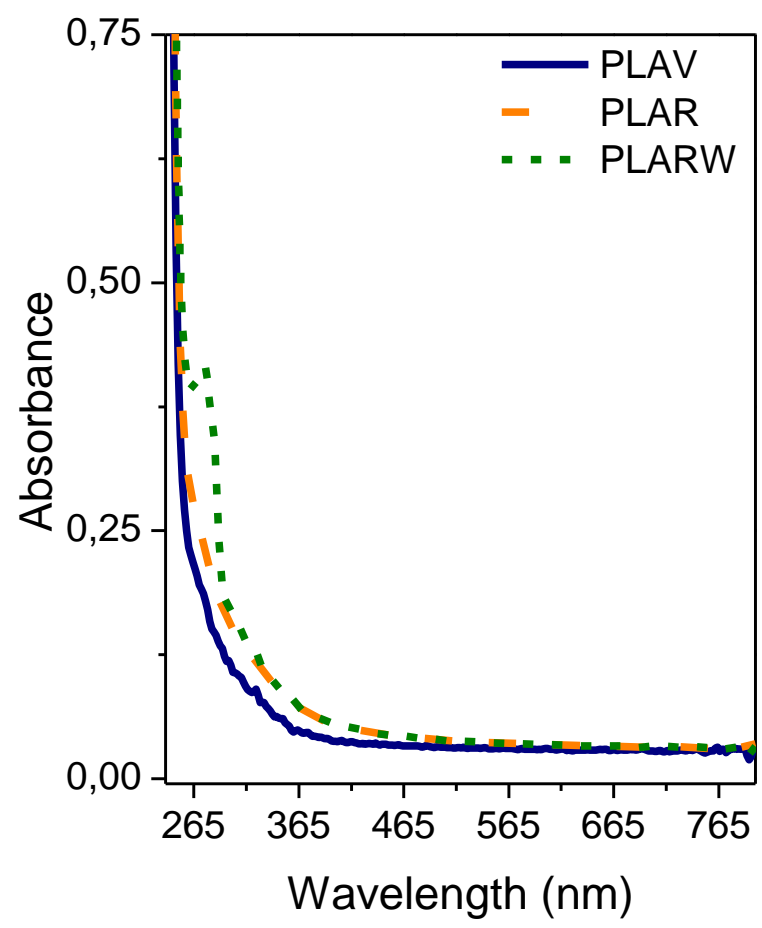

Fig. 6 UV-Vis spectra of PLA subjected to different simulated mechanical recycling processes

Although there are some changes in the UV region of the spectra, the presence of the absorption band located at $270 \mathrm{~nm}$ does not seem to affect the light transmission in the visible region of the spectra. Table 2 presents the percentage of transmitted light between 400 and $800 \mathrm{~nm}$, as well as the haze values for the different materials. It can be seen that both recycled materials have a very good light transmission, greater than $90 \%$, and similar haze values that the virgin polymer. This result points out that despite the reduction in the molecular weight caused by the recycling processes, the optical clarity does not seem to be affected, probably due to the fact that all the materials are amorphous and there are not crystals that could act as reflecting or diffusing centers, which would decrease the optical clarity of PLA. 
Table 2 Haze and light transmission values of virgin and recycled PLA.

\begin{tabular}{ccc}
\hline Sample & Transmission (\%) & Haze \\
\hline PLAV & $92.0 \pm 0.4$ & $2.2 \pm 0.3$ \\
PLAR & $91.1 \pm 0.5$ & $2.8 \pm 0.6$ \\
PLARW & $91.3 \pm 0.8$ & $2.0 \pm 0.2$ \\
\hline
\end{tabular}

\subsubsection{Mechanical properties}

In order to study the effect of the different recycling processes, some mechanical properties were determined by means of instrumented nanoindentation tests, and they are presented on Fig. 7.

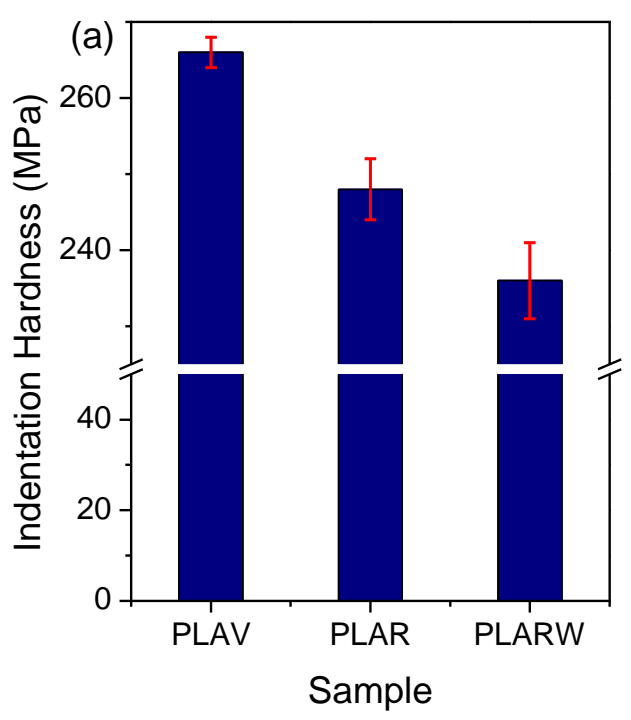




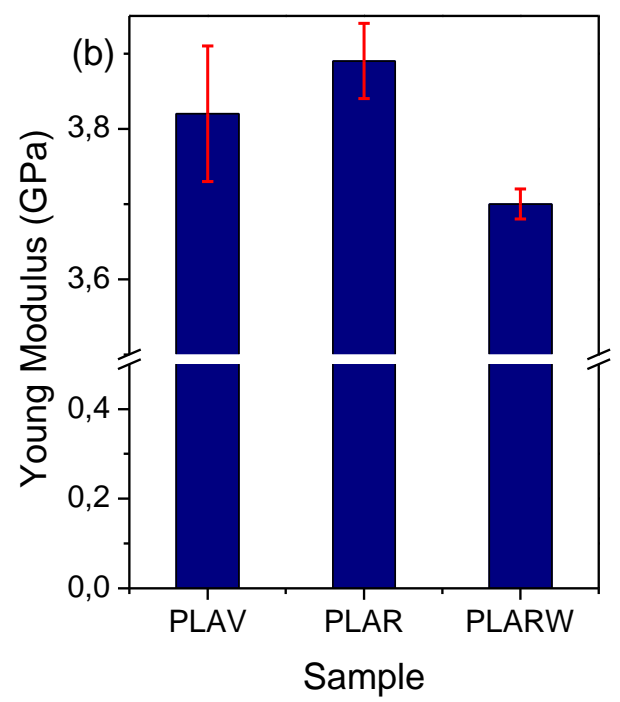

Fig. 7 Indentation hardness (a) and Young modulus (b) of PLA subjected to different simulated mechanical recycling processes

It can be seen on Fig. 7a that the recycled materials present lower hardness values than virgin materials, $\sim 7 \%$ and $\sim 11 \%$ lower for PLAR and PLARW respectively. In the other hand, Fig. 7b shows that the effect of the different recycling processes on the Young modulus is negligible. These results suggest that the decrease in the molecular weight observed in the intrinsic viscosity measurements has a limited effect on the mechanical properties of PLA. In fact, Perego et al. [23] reported that the effect of the molecular weight in the hardness and Young modulus of a multi-reprocessed PLLA is small. As it has been observed by means of UV-Vis spectroscopy, the mechanical recycling led to the generation of new - $\mathrm{COOH}$ groups, which could increase the interaction between the polymer chains, thus counteracting the negative effect of the decrease of molecular weight in the mechanical properties.

\subsubsection{Gas barrier properties}

One of the key properties of the polymers in the field of food packaging is their permeability against different gases. It has been reported by Żenkiewicz et al. [10] that the water vapor and oxygen transmission rates increase by 39 and $18.3 \%$ respectively in a PLA subjected to 10 reprocessing steps. These results highlight the relevance of the studying the effect of the different mechanical recycling processes on the gas barrier properties of PLA. Fig. 8 shows the permeability coefficient of the virgin and recycled PLA for $\mathrm{N}_{2}, \mathrm{O}_{2}$ and $\mathrm{CO}_{2}$. 


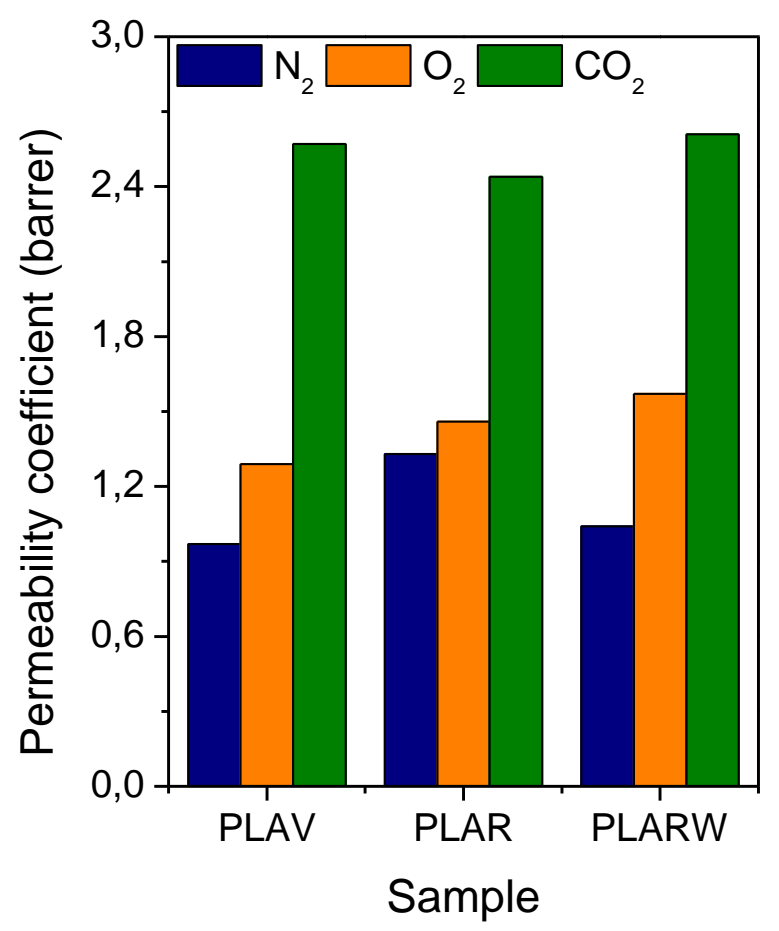

Fig. 8 Permeability coefficient of different gases in virgin and recycled PLA

It can be seen on Fig. 8 that the mechanical recycling does not seem to have a significant effect on the permeability in any of the three gases studied, even for the polymer subjected to the demanding washing step. However, in order to better explain these results, we should consider that, in amorphous polymers, the permeability coefficient is related to the diffusion coefficient and the solubility of the gas in the polymer, according to the following expression [24]:

$P=D x S$

where $P$ is the permeability coefficient, $D$ the diffusion coefficient and $S$ is the gas solubility. Both, $D$ and $S$, depend on several factors, such as the structure of the polymer, the size of the gas molecules, the free volume and temperature, among others. It has been found that for $\mathrm{N}_{2}$ and $\mathrm{O}_{2}$ the diffusion coefficient decreases in the recycled materials, while the solubility increases. The decrease of the diffusion coefficient might be explained by the degradation of the polymer during the recycling processes. The shorter PLA chains present in the recycled materials rearrange themselves better during the physical aging, thus reducing the free volume of the materials and causing the decrease of the diffusion coefficient [24].

On the other hand, the increase of the solubility of $\mathrm{N}_{2}$ and $\mathrm{O}_{2}$ could be related to the presence of more $-\mathrm{COOH}$ groups, which was observed by UV-Vis spectroscopy, as a consequence of the thermomechanical degradation of the polymer during the 
mechanical recycling. The presence of these groups could favor the interactions between the polymer and the gas molecules, which would lead to an increase in the solubility of the gases. Nevertheless, the effects of the thermomechanical degradation on the diffusion coefficient and solubility counteract each other, and the overall effect of the different mechanical recycling processes on the permeability coefficient of PLA is very small, so its use in packaging applications cannot be ruled out.

\section{Conclusions}

We have studied the effect of different mechanical recycling processes on the structure and some important properties, in food packaging applications, of PLA. Three kind of samples were studied, one subjected to a extrusion and compression molding step, one subjected to an accelerated ageing and second extrusion and compression molding step, and one subjected to a demanding washing step prior to the second extrusion and compression molding step. The intrinsic viscosity measurements show that mechanical recycling causes a decrease on the molecular weight of PLA. This effect is more pronounced in the sample subjected to the demanding washing step. Nevertheless, this decrease on the molecular weight is relatively small, of about $20 \%$ in the washed PLA. UV-Vis spectra of the recycled materials show an absorption band at $270 \mathrm{~nm}$, which is related to the presence of $-\mathrm{COOH}$ groups, indicating that thermomechanical degradation during melt processing is responsible for the decrease of the molecular weight of PLA.

The degradation of PLA did not have a significant effect on the structure or degree of crystallinity, as it was show by FTIR-ATR spectroscopy and DSC; however, there was a decrease in cold crystallization temperature as a consequence of the higher mobility of the shorter polymer chains present in the recycled materials.

Regarding the effect of the recycling processes on some properties of PLA, TGA results showed that the thermal stability of PLA is almost unchanged, even in the case of the material subjected to a demanding washing step. This behavior was explained by the generation of compounds with higher thermal stability during the melt reprocessing, counteracting the effect of the decrease of the molecular weight. The lack of changes observed in the degree crystallinity of PLA observed in DSC explains the results obtained by means of UV-Vis spectroscopy and haze measurements, which suggests that recycling processes do not affect the optical clarity of PLA, obtaining light transmission rates above $90 \%$ for all the samples. Furthermore, the decrease of the molecular weight neither seems to have an important effect on the hardness or Young modulus of the different materials, as it was shown by means of nanoindentation tests. Finally, the mechanical recycling process seem to have opposite effects on the diffusion coefficient and solubility of $\mathrm{N} 2$ and $\mathrm{O} 2$ in PLA, while the former decreases during recycling, the latter increases, causing the overall effect of the recycling processes on the permeability to be negligible.

Summarizing, the obtained indicate that the moderate degradation of PLA during its use and mechanical recycling has a limited impact on the structure, thermal stability, mechanical, optical and gas barrier properties. Therefore, the results reveal the potential of mechanically recycled PLA to be reused, even in demanding applications such as food packaging. 


\section{Acknowledgements}

We acknowledge the financial support of MINECO-Spain (project MAT201347972-C2-2-P), project UPM RP 160543006 and Ecoembes.

\section{References}

1. Reddy, M.M., Vivekanandhan, S., Misra, M., Bhatia, S.K., Mohanty, A.K.: Biobased plastics and bionanocomposites: Current status and future opportunities. Prog. Polym. Sci. 38, 1653-1689 (2013)

2. Auras, R., Lim, L., Selke, S.E.M., Tsuji, H. : Poly(Lactic Acid): Synthesis, Structures, Properties, Processing, and Applications. John Wiley \& Sons, Inc., (2010)

3. Aeschelmann, F., Carus, M.: Biobased Building Blocks and Polymers in the World: Capacities, Production, and Applications-Status Quo and Trends Towards 2020. Ind. Biotechnol. 11, 154-159 (2015)

4. Mülhaupt, R.: Green Polymer Chemistry and Bio-based Plastics: Dreams and Reality. Macromol. Chem. Phys. 214, 159-174 (2013)

5. Niaounakis, M. : Biopolymers Reuse, Recycling, and Disposal. William Andrew Publishing, Oxford (2013)

6. Piemonte, V.: Bioplastic Wastes: The Best Final Disposition for Energy Saving. J. Polym. Environ. 19, 988-994 (2011)

7. Cornell, D.D.: Biopolymers in the Existing Postconsumer Plastics Recycling Stream. J. Polym. Environ. 15, 295-299 (2007)

8. Soroudi, A., Jakubowicz, I.: Recycling of bioplastics, their blends and biocomposites: A review. Eur. Polym. J. 49, 2839-2858 (2013)

9. Pillin, I., Montrelay, N., Bourmaud, A., Grohens, Y.: Effect of thermo-mechanical cycles on the physico-chemical properties of poly(lactic acid). Polym. Degrad. Stab. 93, 321-328 (2008)

10. Żenkiewicz, M., Richert, J., Rytlewski, P., Moraczewski, K., Stepczyńska, M., Karasiewicz, T.: Characterisation of multi-extruded poly(lactic acid). Polym. Test. 28, 412-418 (2009)

11. Nascimento, L., Gamez-Perez, J., Santana, O.O., Velasco, J.I., Maspoch, M.L., Franco-Urquiza, E.: Effect of the Recycling and Annealing on the Mechanical and Fracture Properties of Poly(Lactic Acid). J. Polym. Environ. 18, 654-660 (2010)

12. Kister, G., Cassanas, G., Vert, M.: Effects of morphology, conformation and configuration on the IR and Raman spectra of various poly(lactic acid)s. Polymer 39, 267-273 (1998)

13. Lim, L.-., Auras, R., Rubino, M.: Processing technologies for poly(lactic acid). Prog. Polym. Sci. 33, 820-852 (2008) 
14. Le Marec, P.E., Ferry, L., Quantin, J., Bénézet, J., Bonfils, F., Guilbert, S., Bergeret, A.: Influence of melt processing conditions on poly(lactic acid) degradation: Molar mass distribution and crystallization. Polym. Degrad. Stab. 110, 353-363 (2014)

15. Badia, J.D., Strömberg, E., Karlsson, S., Ribes-Greus, A.: Material valorisation of amorphous polylactide. Influence of thermo-mechanical degradation on the morphology, segmental dynamics, thermal and mechanical performance. Polym. Degrad. Stab. 97, 670-678 (2012)

16. Chariyachotilert, C., Joshi, S., Selke, S.E.M., Auras, R.: Assessment of the properties of poly(L-lactic acid) sheets produced with differing amounts of postconsumer recycled poly(L-lactic acid). J. Plast. Film Sheeting 28, 314-335 (2012)

17. Badia, J.D., Santonja-Blasco, L., Martínez-Felipe, A., Ribes-Greus, A.: Hygrothermal ageing of reprocessed polylactide. Polym. Degrad. Stab. 97, 1881-1890 (2012)

18. Scaffaro, R., Morreale, M., Mirabella, F., La Mantia, F.P.: Preparation and Recycling of Plasticized PLA. Macromol. Mater. Eng. 296, 141-150 (2011)

19. Di Lorenzo, M.L.: Calorimetric analysis of the multiple melting behavior of poly(Llactic acid). J. Appl. Polym. Sci. 100, 3145-3151 (2006)

20. Yasuniwa, M., Tsubakihara, S., Sugimoto, Y., Nakafuku, C.: Thermal analysis of the double-melting behavior of poly(L-lactic acid). J. Polym. Sci. B Polym. Phys. 42, 2532 (2004)

21. Carrasco, F., Pagès, P., Gámez-Pérez, J., Santana, O.O., Maspoch, M.L.: Processing of poly(lactic acid): Characterization of chemical structure, thermal stability and mechanical properties. Polym. Degrad. Stab. 95, 116-125 (2010)

22. Badía, J.D., Strömberg, E., Ribes-Greus, A., Karlsson, S.: Assessing the MALDITOF MS sample preparation procedure to analyze the influence of thermo-oxidative ageing and thermo-mechanical degradation on poly (Lactide). Eur. Polym. J. 47, 1416$1428(2011)$

23. Perego, G., Cella, G.D., Bastioli, C.: Effect of molecular weight and crystallinity on poly(lactic acid) mechanical properties. J. Appl. Polym. Sci. 59, 37-43 (1996)

24. Choudalakis, G., Gotsis, A.D.: Permeability of polymer/clay nanocomposites: A review. Eur. Polym. J. 45, 967-984 (2009) 